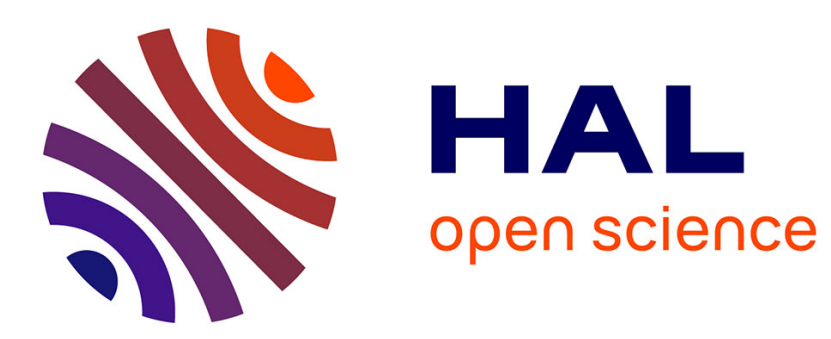

\title{
Optimization of the antenna array geometry based on a Bayesian DOA estimation criterion
}

\author{
Houcem Gazzah, Jean-Pierre Delmas
}

\section{To cite this version:}

Houcem Gazzah, Jean-Pierre Delmas. Optimization of the antenna array geometry based on a Bayesian DOA estimation criterion. ICASSP 2011: 36th International Conference on Acoustics, Speech and Signal Processing, May 2011, Prague, Czech Republic. pp.2544-2547, 10.1109/ICASSP.2011.5947003 . hal-01302427

\section{HAL Id: hal-01302427 \\ https://hal.science/hal-01302427}

Submitted on 14 Apr 2016

HAL is a multi-disciplinary open access archive for the deposit and dissemination of scientific research documents, whether they are published or not. The documents may come from teaching and research institutions in France or abroad, or from public or private research centers.
L'archive ouverte pluridisciplinaire HAL, est destinée au dépôt et à la diffusion de documents scientifiques de niveau recherche, publiés ou non, émanant des établissements d'enseignement et de recherche français ou étrangers, des laboratoires publics ou privés. 


\section{OPTIMIZATION OF THE ANTENNA ARRAY GEOMETRY BASED ON A BAYESIAN DOA ESTIMATION CRITERION}

\author{
Houcem Gazzah \\ Dept. of Elec. and Computer Engineering \\ University of Sharjah, 27272, UAE \\ hgazzah@sharjah.ac.ae
}

\author{
Jean-Pierre Delmas \\ Telecom SudParis, UMR CNRS 5157 \\ 91011 Evry, France \\ jean-pierre.delmas@it-sudparis.eu
}

\begin{abstract}
In this paper, we address the problem of the sensor placement for estimating the direction of a narrow-band source, randomly located in the far-field of a planar antenna array. Estimation performance is evaluated by means of the expectation of the conditional Cramer Rao bound (ECRB), which depends on the prior probabilistic distribution of the DOA angles. We study the particular, but practical, case where the azimuth angle is uniformly distributed. Surprisingly, it turns out that the optimal arrays are not isotropic, i.e. they do not have the same accuracy in all possible look directions. In fact, optimal arrays computed here increase performance by about $10 \%$ compared to optimal isotropic arrays computed in a previous work.
\end{abstract}

Index Terms - Direction of arrival estimation

\section{INTRODUCTION}

Estimation of the Direction of Arrival (DOA) is a major topic in signal processing with connections to many important applications. Despite a long research effort, the sensor placement problem has often been overlooked, with only a few known facts about the impact of the array geometry on the estimation performance $[1,2,3,4]$. One reason is a sophisticated expression of the Cramer-Rao bound (CRB), even in the single source case [5]. An other reason is the antenna ambiguity problem [6] that translates into a set of sophisticated constraints. Recently, the CRB in [5] was shown to be a simple sinusoidal function of the azimuth and elevation angles [7]. Later, by imposing an ambiguity-free curve-like array structure, unconstrained optimization problems have been developed and solved numerically [8]. The most interesting outcome of this optimization was the so-called isotropic antenna array, that exhibits the same accuracy (in terms of the CRB) regardless of the actual source location [9]. Optimum isotropic array geometries have been found that were not only more accurate [the associated CRB, on both azimuth and elevation, are reduced by as much as $29 \%$ compared to Uniform Circular Arrays (UCA)], but also non-intuitive, because they have a V-like geometry that has no central-symmetry.
The present paper is an adaptation of ideas from $[7,8]$ to a different context. Instead of having a fixed (though unknown) position, the source is randomly located in the antenna far-field. Instead of being characterized by the DOA (azimuth and elevation ) angles, its position is rather characterized by a prior probabilistic distribution of these angles. A new optimization criterion is subsequently derived, using elements from the Bayesian estimation theory [10] and solved while imposing an ambiguity-free array structure and assuming a uniformly distributed source (azimuth) angle of arrival. The outcome of the optimization procedure are, once again, concave curve-like arrays. Surprisingly enough, optimum antenna arrays for a prior uniformly distributed DOA are not isotropic. Hence, the newly obtained optimum arrays are able to outperform those obtained in [9] to achieve a larger $36 \%$ reduction of the (Bayesian) CRB, compared to the UCA.

\section{DATA MODEL AND PREVIOUS RESULTS}

A source is emitting a narrow-band signal $s(t)$ that is Gaussian zero-mean distributed, centered at frequency $c / \lambda$. A planar antenna array is made of $M$ identical and omni-

directional sensors placed in the $(x, y)$ plane. As shown in Fig. 1, the position of the $m$-th sensor is given by the polar coordinates $\rho_{m}$ and $\phi_{m}$ or, equivalently, by the complex number $\gamma_{m} \hat{=} \rho_{m} \exp \left(j \phi_{m}\right)$. A source DOA located in the antenna far-field is characterized by its angles: the azimuth angle $\Phi$ and the elevation angle $\Theta$. If $x_{m}(t), m=1, \cdots, M$, is the snapshot induced by the source at time index $t$ at the $m$-th sensor, then $\mathbf{x}(t) \hat{=}\left[x_{1}(t) \cdots x_{M}(t)\right]^{T}$ stands for the $M$ dimensional array output. The observation is the following

$$
\mathbf{x}(t)=\left[\begin{array}{c}
\exp \left[2 j \pi \frac{\rho_{1}}{\lambda} \sin (\Theta) \cos \left(\Phi-\phi_{1}\right)\right] \\
\vdots \\
\exp \left[2 j \pi \frac{\rho_{M}}{\lambda} \sin (\Theta) \cos \left(\Phi-\phi_{M}\right)\right]
\end{array}\right] s(t)+\mathbf{n}(t)
$$

where $\mathbf{n}(t) \hat{=}\left[n_{1}(t), \cdots, n_{M}(t)\right]^{T}$ denotes an additive noise that is Gaussian, zero-mean distributed whose components on the different sensors are assumed to be mutually independent. The different snapshots $\mathbf{x}(t)$ are independent and identically 


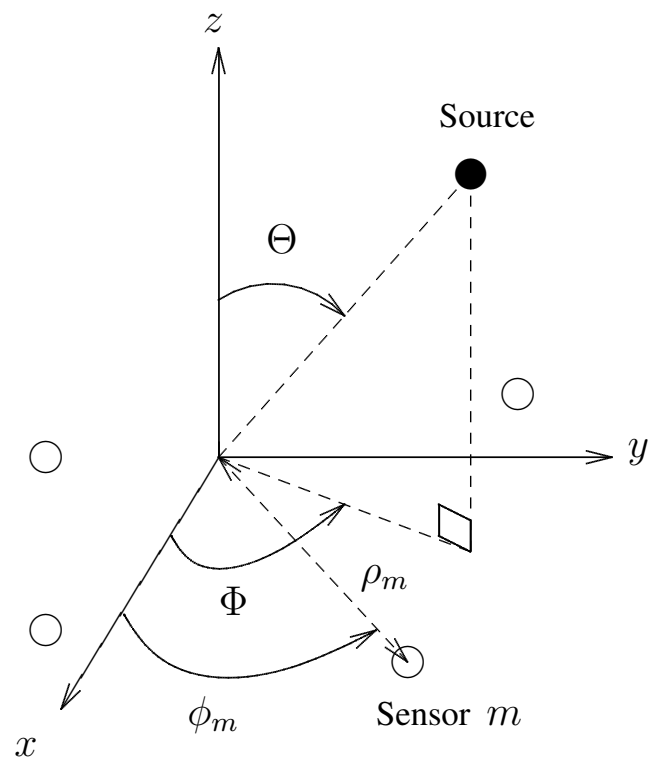

Fig. 1. Planar array and source DOAs.

distributed. We denote $\sigma_{s}^{2} \hat{=} \mathrm{E}\left[|s(t)|^{2}\right]$ and $\sigma_{n}^{2} \hat{=} \mathrm{E}\left[\left|n_{m}(t)\right|^{2}\right]$ for all $m$. Based on $N$ snapshots $\mathbf{x}\left(t_{1}\right), \cdots, \mathbf{x}\left(t_{N}\right)$, a variety of techniques, notably the MUSIC algorithm [11] can estimate the angle $(\Phi, \Theta)$ with a variance that attains asymptotically (with respect to $N$ ) the lowest MSE achievable by an unbiased estimator, the so-called CRB [5].

Under the above assumptions, the CRB associated with the unknown deterministic parameters $\left(\sigma_{s}^{2}, \sigma_{n}^{2}, \Phi, \Theta\right)$ has been concentrated on the DOA parameters only [5, rel.(39)] and has been proven [7] to have a very simple and attractive sinusoidal form:

$$
\mathbf{C}=\left[\begin{array}{ll}
\mathbf{C}_{\Phi \Phi} & \mathbf{C}_{\Phi \Theta} \\
\mathbf{C}_{\Theta \Phi} & \mathbf{C}_{\Theta \Theta}
\end{array}\right]
$$

with

$$
\begin{aligned}
\mathbf{C}_{\Phi \Phi} & =\frac{A}{\sin ^{2}(\Theta)} B(\Phi) \\
\mathbf{C}_{\Theta \Theta} & =\frac{A}{\cos ^{2}(\Theta)} B\left(\Phi+\frac{\pi}{2}\right) \\
\mathbf{C}_{\Phi \Theta} & =\frac{A}{\sin (2 \Theta)} \frac{\Im\left[S_{1} \exp (-2 j \Phi)\right]}{\left|S_{1}\right|^{2}-S_{0}^{2}}
\end{aligned}
$$

where $A \hat{=}\left[1+\sigma_{n}^{2} /\left(M \sigma_{s}^{2}\right)\right]\left[\sigma_{n}^{2} /\left(4 \pi^{2} N \sigma_{s}^{2}\right)\right]$ only depends on the observation SNR, the antenna size $M$ and the number of snapshots $N$, and

$$
B(\Phi) \hat{=} \frac{S_{0}+\Re\left[S_{1} \exp (-2 j \Phi)\right]}{S_{0}^{2}-\left|S_{1}\right|^{2}}
$$

is a function of the source azimuth angle and the array- geometry dependent constants

$$
\begin{aligned}
& S_{0} \hat{=} \sum_{m=1}^{M}\left|\frac{\gamma_{m}}{\lambda}\right|^{2}-\frac{1}{M}\left|\sum_{m=1}^{M} \frac{\gamma_{m}}{\lambda}\right|^{2} \\
& S_{1} \hat{=} \sum_{m=1}^{M}\left(\frac{\gamma_{m}}{\lambda}\right)^{2}-\frac{1}{M}\left(\sum_{m=1}^{M} \frac{\gamma_{m}}{\lambda}\right)^{2} .
\end{aligned}
$$

Obviously, an antenna array for which $S_{1}=0$ has a constant CRB regardless of the source actual (azimuth) angle, and, for this reason, is referred to as isotropic. UCA is an example of such an antenna.

\section{AN OPTIMIZATION CRITERION FOR THE RANDOMLY LOCATED SOURCE}

We assume that the source is randomly located in the space, with $(\Phi, \Theta)$ following some known prior joint distribution. In this context, lower bounds on the MSE matrix associated with an arbitrary estimate $(\widehat{\Phi}, \widehat{\Theta})$

$$
\mathbf{R}(\widehat{\Phi}, \widehat{\Theta})=\left[\begin{array}{cc}
\mathrm{E}(\widehat{\Phi}-\Phi)^{2} & \mathrm{E}(\widehat{\Phi}-\Phi)(\widehat{\Theta}-\Theta) \\
\mathrm{E}(\widehat{\Theta}-\Theta)(\widehat{\Phi}-\Phi) & \mathrm{E}(\widehat{\Theta}-\Theta)^{2}
\end{array}\right] .
$$

can be given [10]. In particular $\mathbf{R}(\widehat{\Phi}, \widehat{\Theta})$ is lower bounded by the celebrated Bayesian Cramer Rao bound (BCRB). Because this bound is based on the expectation of the Fisher information matrix, i.e., the inverse of (1), we prefer to use the expectation of the conditional Cramer Rao bound (ECRB) with is simpler to derive [10, rel.(10)]

$$
\overline{\mathbf{C}} \hat{=} \mathrm{E}_{\Phi, \Theta}(\mathbf{C})=\left[\begin{array}{ll}
\overline{\mathbf{C}}_{\Phi \Phi} & \overline{\mathbf{C}}_{\Phi \Theta} \\
\overline{\mathbf{C}}_{\Theta \Phi} & \overline{\mathbf{C}}_{\Theta \Theta}
\end{array}\right] \text {. }
$$

This lower bound is asymptotically (with respect to $N$ ) attained by the MSE of the maximum a posteriori (MAP) estimate $[10$, rel. (37)]. But we note in contrast, that the BCRB may not be a tight lower bound asymptotically because here, CRB (1) depends on $(\Phi, \Theta)$ [10, rel.(39)].

If we further assume independent azimuth and elevation prior distributions with respective $\operatorname{PDF} f(\Phi)$ and $g(\Theta)$, we have

$$
\begin{aligned}
\overline{\mathbf{C}}_{\Phi \Phi} & =A \int_{-\infty}^{\infty} \frac{g(\Theta) d \Theta}{\sin ^{2}(\Theta)} \int_{-\infty}^{\infty} B(\Phi) f(\Phi) d \Phi \\
\overline{\mathbf{C}}_{\Theta, \Theta} & =A \int_{-\infty}^{\infty} \frac{g(\Theta) d \Theta}{\cos ^{2}(\Theta)} \int_{-\infty}^{\infty} B\left(\Phi+\frac{\pi}{2}\right) f(\Phi) d \Phi .
\end{aligned}
$$

We compare to the $M$-sized UCA with inter-sensor spacing $d$ for which $B^{\prime} \hat{=} B(\Phi)=4 \lambda^{2} \sin ^{2}(\pi / M) /\left(M d^{2}\right)$. Given the above, and letting $\varphi_{\Phi}$ be the characteristic function relative to the random parameter $\Phi$, we prove that

$$
\begin{aligned}
\frac{\overline{\mathbf{C}}_{\Phi \Phi}}{\left.\overline{\mathbf{C}}_{\Phi \Phi}\right|_{\mathrm{UCA}}} & =\frac{1}{B^{\prime}} \frac{S_{0}+\Re\left[S_{1} \varphi_{\Phi}(-2)\right]}{S_{0}^{2}-\left|S_{1}\right|^{2}} \\
\frac{\overline{\mathbf{C}}_{\Theta \Theta}}{\left.\overline{\mathbf{C}}_{\Theta \Theta}\right|_{\mathrm{UCA}}} & =\frac{1}{B^{\prime}} \frac{S_{0}-\Re\left[S_{1} \varphi_{\Phi}(-2)\right]}{S_{0}^{2}-\left|S_{1}\right|^{2}}
\end{aligned}
$$




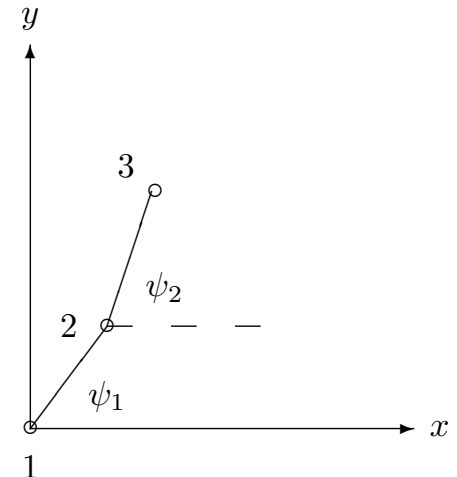

Fig. 2. Sensors numbering and angular parameters $\psi_{m}$.

The above will serve as our performance measures, advantageously independent from the distribution of the elevation angle.

\section{AMBIGUITY-FREE ARRAY STRUCTURE}

As depicted in Fig. 2, for a planar array in the $(x, y)$ plane, we assume the first sensor to be placed at the origin. To ensure a minimal inter-sensors distance $d$ (practically fixed to $\lambda / 2$ ), and guarantee an array exempt from (first-order) ambiguities [6, Th. 9], sensors are placed such that for all $m \geq 2, \mid \gamma_{m}-$ $\gamma_{m-1} \mid=d$ or also

$$
\frac{\gamma_{m}}{d}=\frac{\gamma_{m-1}}{d}+\exp \left(j \psi_{m-1}\right)
$$

At the same time, the so-structured array is now more conveniently characterized by a set of bounded parameters $\psi_{1}, \cdots, \psi_{M-1}$, each in $[-\pi, \pi[$.

We introduce $B^{\prime \prime} \hat{=} d^{2} B^{\prime} / \lambda^{2}=4 \sin ^{2}(\pi / M) / M$ and

$$
\begin{aligned}
& T_{0} \hat{=} \frac{\lambda^{2}}{d^{2}} S_{0}=\sum_{m=1}^{M} \frac{\left|\gamma_{m}\right|^{2}}{d^{2}}-\frac{1}{M}\left|\sum_{m=1}^{M} \frac{\gamma_{m}}{d}\right|^{2} \\
& T_{1} \hat{=} \frac{\lambda^{2}}{d^{2}} S_{1}=\sum_{m=1}^{M} \frac{\gamma_{m}^{2}}{d^{2}}-\frac{1}{M}\left(\sum_{m=1}^{M} \frac{\gamma_{m}}{d}\right)^{2},
\end{aligned}
$$

both of them depend on $\psi_{1}, \cdots, \psi_{M-1}$ only. One can easily prove that

$$
\begin{aligned}
\frac{\overline{\mathbf{C}}_{\Phi \Phi}}{\left.\overline{\mathbf{C}}_{\Phi \Phi}\right|_{\mathrm{UCA}}} & =\frac{1}{B^{\prime \prime}} \frac{T_{0}+\Re\left[T_{1} \varphi_{\Phi}(-2)\right]}{T_{0}^{2}-\left|T_{1}\right|^{2}} \\
\frac{\overline{\mathbf{C}}_{\Theta \Theta}}{\left.\overline{\mathbf{C}}_{\Theta \Theta}\right|_{\mathrm{UCA}}} & =\frac{1}{B^{\prime \prime}} \frac{T_{0}-\Re\left[T_{1} \varphi_{\Phi}(-2)\right]}{T_{0}^{2}-\left|T_{1}\right|^{2}}
\end{aligned}
$$

The optimization problem is, hence, made independent from the actual inter-sensor placement.

\section{UNIFORMLY DISTRIBUTED AZIMUTH}

For uniformly prior distributed azimuth angle,

$$
\varphi_{\Phi}(-2)=\mathrm{E}[\exp (-2 j \Phi)]=0,
$$

leading to a unique performance criterion

$$
\frac{\overline{\mathbf{C}}_{\Phi \Phi}}{\left.\overline{\mathbf{C}}_{\Phi \Phi}\right|_{\mathrm{UCA}}}=\frac{\overline{\mathbf{C}}_{\Theta \Theta}}{\left.\overline{\mathbf{C}}_{\Theta \Theta}\right|_{\mathrm{UCA}}}=\frac{1}{\mathbf{B}^{\prime}} \frac{T_{0}}{T_{0}^{2}-\left|T_{1}\right|^{2}}
$$

where $B^{\prime}$ does not depend on the array geometry. Consequently, the optimization problem consists in minimizing $T_{0} /\left(T_{0}^{2}-\left|T_{1}\right|^{2}\right)$ i.e. the unconstrained maximization of

$$
T_{0}-\frac{\left|T_{1}\right|^{2}}{T_{0}}
$$

Contrarily to an intuition, the optimal array for a uniformly located source does not have to be isotropic. If $T_{1}=0$, (2) and (3) implying that (4) is verified by isotropic arrays. Nevertheless, it is minimized by a non-isotropic one, as will be confirmed by the exhaustive search.

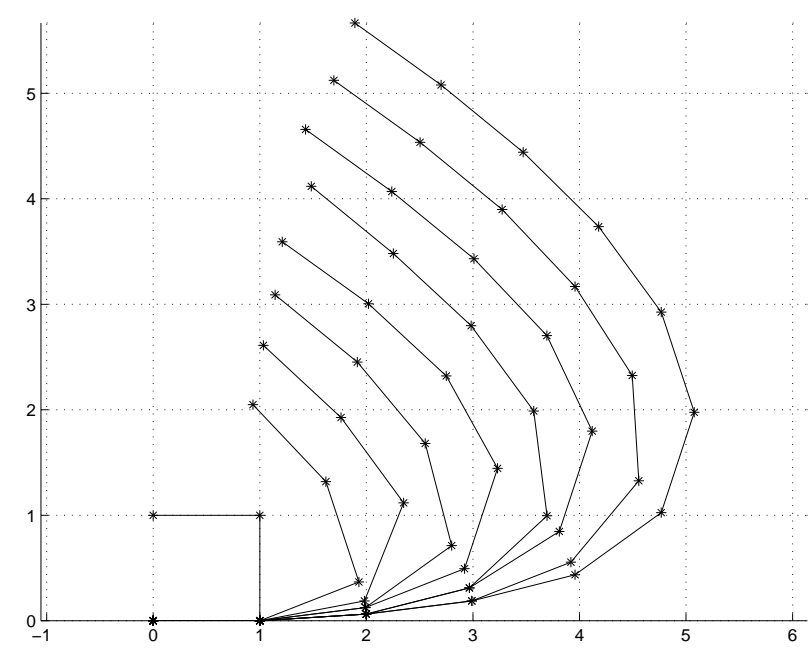

(a)

Fig. 3. Optimal antenna arrays with $M=4, \cdots, 12$ sensors, obtained by systematic search for a uniformly prior distributed azimuth angle.

Without loss of generality, we can impose $\psi_{1}=0\left(T_{0}\right.$ and $\left|T_{1}\right|$ are unchanged if the array is rotated) and $\psi_{2} \in[0, \pi]$ ( $T_{0}$ and $\left|T_{1}\right|$ are unchanged if the array is replaced by the one obtained by $x$-axis symmetry). We are left to determine, by means of an exhaustive search, parameters $\psi_{2}, \psi_{3}, \cdots$ that maximize (5). The resulting optimal arrays are shown in Fig. 3. None of them is isotropic ( $T_{1}$ is non-zero), except when $M=4$ (optimal array is the UCA). The optimum 


\begin{tabular}{|r||c|c|c|c|}
\hline$M$ & 5 & 6 & 7 & 8 \\
\hline \hline Opt. & 0.7306 & 0.7039 & 0.6805 & 0.6689 \\
\hline Opt. isot. & 0.8075 & 0.8005 & 0.7617 & 0.7566 \\
\hline \hline$M$ & 9 & 10 & 11 & 12 \\
\hline \hline Opt. & 0.6597 & 0.654 & 0.6494 & 0.6461 \\
\hline Opt. isot. & 0.7441 & 0.7398 & 0.7331 & 0.7302 \\
\hline \hline
\end{tabular}

Table 1. Normalized CRBs of optimal arrays [maximizing $T_{0}-$ $\left|T_{1}\right|^{2} / T_{0}$, i.e. (5)] and of optimal isotropic arrays (maximizing $T_{0}$ under $T_{1}=0$ [8]). Arrays with 9 or more sensors have been computed assuming a concave array geometry.

isotropic ones have been computed in [8] and their performance is given in Tab. 1 comparatively to the optimum arrays computed here. It is clear that a significant improvement is obtained by relaxing the condition $T_{1}=0$. This justifies the interest of the analysis given in this paper which is leading to the non-trivial conclusion that the optimal array for a source that is uniformly prior distributed around the array is not isotropic.

The complexity of the systematic search is exponential and becomes unpractical for large array sizes (adding one more sensor means that the grid size is multiplied by 50 in our computations). At the same time, we notice, from the obtained results, that the array has a concave geometry. We implement this feature into the search procedure so that larger array sizes can be investigated while maintaining a small search step ( $\pi / 50$ in our computations). A concave array is one that verifies

$$
\psi_{m} \geq \psi_{m-1} \text { for } m \geq 2 .
$$

Optimal isotropic antenna arrays with sizes $M \geq 9$ are computed using the so-modified search procedure. The obtained optimal arrays are plotted in Fig. 3, and their performance, expressed in terms of the normalized CRB, are shown in Tab. 1. As a conclusion, as the antenna size increases, optimal arrays show a normalized $\mathrm{CRB}$ equal to 0.64 , compared to 0.71 for optimal isotropic arrays from [9].

\section{CONCLUSION}

By using tools from the Bayesian estimation theory, we express the ECRB as function of the prior probabilistic distribution of the DOA angles of a randomly located source. A geometry-dependent performance measure is subsequently defined and optimized in the special case of a uniformly distributed azimuth angle. Optimal arrays are computed and turn to be different from optimal isotropic arrays computed in $[8,9]$. They reduce the ECRB by $36 \%$ compared to UCA, and by $10 \%$ compared to optimal isotropic arrays in $[8,9]$. Extension of this work to other specific a priori distributions of $(\Phi, \Theta)$ is underway.

\section{REFERENCES}

[1] A. Mirkin and L. H. Sibul, "Cramér-Rao bounds on angle estimation with a two-dimensional array," IEEE Trans. Signal Process., vol. 39, no. 2, pp. 515-517, Feb. 1991.

[2] R. O. Nielsen "Azimuth and elevation angle estimation with a three-dimensional array," IEEE J. Ocean. Eng., vol. 19, no. 1, pp. 84-86, Jan. 1994.

[3] M. Hawkes and A. Nehorai "Effects of sensor placement on acoustic vector-sensor array performance," IEEE J. Ocean. Eng., vol. 24, no. 1, pp. 33-40, Jan. 1999.

[4] Ü. Baysal and R. L. Moses, "On the geometry of isotropic arrays," IEEE Trans. Signal Process., vol. 51, no.6, pp. 1469-1478, Jun. 2003.

[5] B. Porat and B. Friedlander, "Analysis of the asymptotic relative efficiency of the MUSIC algorithm," IEEE Trans. Acoust., Speech, Signal Process., vol. 36, no. 4, pp. 532544, Apr. 1988.

[6] L. C. Godara and A. Cantoni, "Uniqueness and linear independence of steering vectors in array space," J. Acoust. Soc. Amer., vol. 70, no. 2, pp. 467-475, Aug. 1981.

[7] H. Gazzah and S. Marcos, "Cramer-Rao bounds for antenna array design," IEEE Trans. Signal Process., vol. 54, no. 1, pp. 336-345, Jan. 2006.

[8] H. Gazzah and K. Abed-Meraim "Optimum AmbiguityFree Directional and Omni-Directional Planar Antenna Arrays for DOA Estimation," IEEE Trans. Signal Process., vol. 57, no. 10, pp. 3942-3253, Oct. 2009.

[9] H. Gazzah, "Optimum Antenna Arrays for Isotropic Direction Finding," IEEE Trans. Aerosp. Electron. Syst., to be published.

[10] H. L. Van Trees and K. L. Bell, Bayesian Bounds for Parameter Estimation and Nonlinear Filtering/Tracking, H. L. Van Trees and K. L. Bell, Ed. John Wiley \& Sons, 2007.

[11] R. O. Schmidt, "Multiple emitter location and signal parameter estimation," IEEE Trans. Antennas Propagat., vol. AP-34, no. 3, pp. 276-280, Mar. 1986. 